\title{
FEATURE TRACKING FOR VENTRICULAR STRAIN ASSESSMENT IN HEART FAILURE WITH PRESERVED EJECTION FRACTION
}

\author{
Liang Zhong ${ }^{1,2}$, Shuang Leng ${ }^{1}$, Xiao-Dan Zhao ${ }^{1}$, Ru-San Tan ${ }^{1,2}$ \\ ${ }^{1}$ National Heart Centre Singapore, 5 Hospital Drive, Singapore 169609 \\ ${ }^{2}$ Duke-NUS Medical School, 8 College Road, Singapore 169857
}

Impairment of left ventricular (LV) longitudinal function is recognized as an independent predictor of cardiac events in patients with heart failure (HF). ${ }^{1}$ Strain imaging derived from speckle tracking echocardiography or feature tracking cardiovascular magnetic resonance (CMR $)^{2}$ permit assessments of myocardial function in the longitudinal direction, however, specific competencies and time-consuming protocols are often needed. Therefore, we aim to investigate a fast, semi-automated, and vendor-independent approach for accurate determination of LV longitudinal strain from standard cine CMR images.

The LV longitudinal strain parameters are measured using a semi-automatic post-processing algorithm that tracked, on standard two- and four-chamber cine CMR views, the distance ( $L$ ) between the left atrioventricular junction and apical epicardium (Fig. 1). The atrioventricular junctions are selected as the mitral valve insertion points at the septal and lateral borders of the annulus on the four-chamber view, and the anterior and inferior annular insertion points on the two-chamber view. ${ }^{3}$ The strain of each wall at any time point $(t)$ in the cardiac cycle from LV end-diastole (ED, time 0 ) is calculated based on the strain formula: $(L(t)-L(0)) \times 100 / L(0)$. Strain rate is derived by taking the first-order derivative of the strain. The peak systolic longitudinal strain is obtained at $t$ equal to LV end-systole (ES), and the corresponding peak systolic strain rate is obtained from the strain rate curve. All resulting values from the 4 walls in two- and four-chamber views are averaged to obtain the global longitudinal strain (GLS) and global longitudinal strain rate (GLSR) of the whole LV. The fast GLS assessment was successfully performed in 50 normal controls, and $100 \mathrm{HF}$ patients of which 30 had preserved ejection fraction (HFpEF, LVEF $\geq 50 \%$ ); 30 had mid-range EF (HFmrEF, LVEF $40-49 \%$ ); and 40 had reduced EF (HFrEF, LVEF < 40\%). Processing time averaged $76 \pm 9$ seconds per case. Intra- and inter-observer variations were $3.3 \%-5.8 \%$ (all intra-class correlation coefficient $>0.9$ ). Significantly reduced LV GLS and GLSR were observed in all patient groups compared to normal controls. Patients with HFpEF and HFmrEF had significantly larger LV GLS and GLSR than HFrEF, while there were no significant differences between HFpEF and HFmrEF (Table 1). On multivariate analysis, fast LV GLS was found to be an independent predictor of HFpEF and HFmrEF. The area under the receiver operating characteristic curve in detecting HFpEF and HFmrEF from normal controls (GLS < 15.0\%) was 0.958 (sensitivity $=93 \%$, specificity $=86 \%$ ). The presented fast LV strain measurements represent reliable and expeditiously calculable parameters for quantifying longitudinal LV deformation using routine clinical cine CMR images without specific acquisition protocol or special software tools.

\section{References:}

1. Zou H, Xi C, Zhao XD, Koh AS, Gao F, Su Y, Tan RS, Allen J, Lee LC, Genet M, Zhong L. Quantification of biventricular strains in heart failure with preserved ejection fraction patients using hyperelastic warping method. Front Physiol. 2018;9:1295.

2. Peng JP, Zhao XD, Zhao L, Fan Z, Wang Z, Cheng H, Leng S, Allen J, Tan RS, Koh AS, Ma XH, Lou M, Zhong L. Normal values of myocardial deformation assessed by cardiovascular magnetic resonance feature tracking in a healthy Chinese population: A multicenter study. Front Physiol. 2018;9:1181.

3. Leng S, Tan RS, Zhao XD, Allen J, Koh AS, Zhong L. Validation of a rapid semi-automated method to assess left atrial longitudinal phasic strains on cine cardiovascular magnetic resonance imaging. J Cardiovasc Magn Reson. 2018;20:71. 

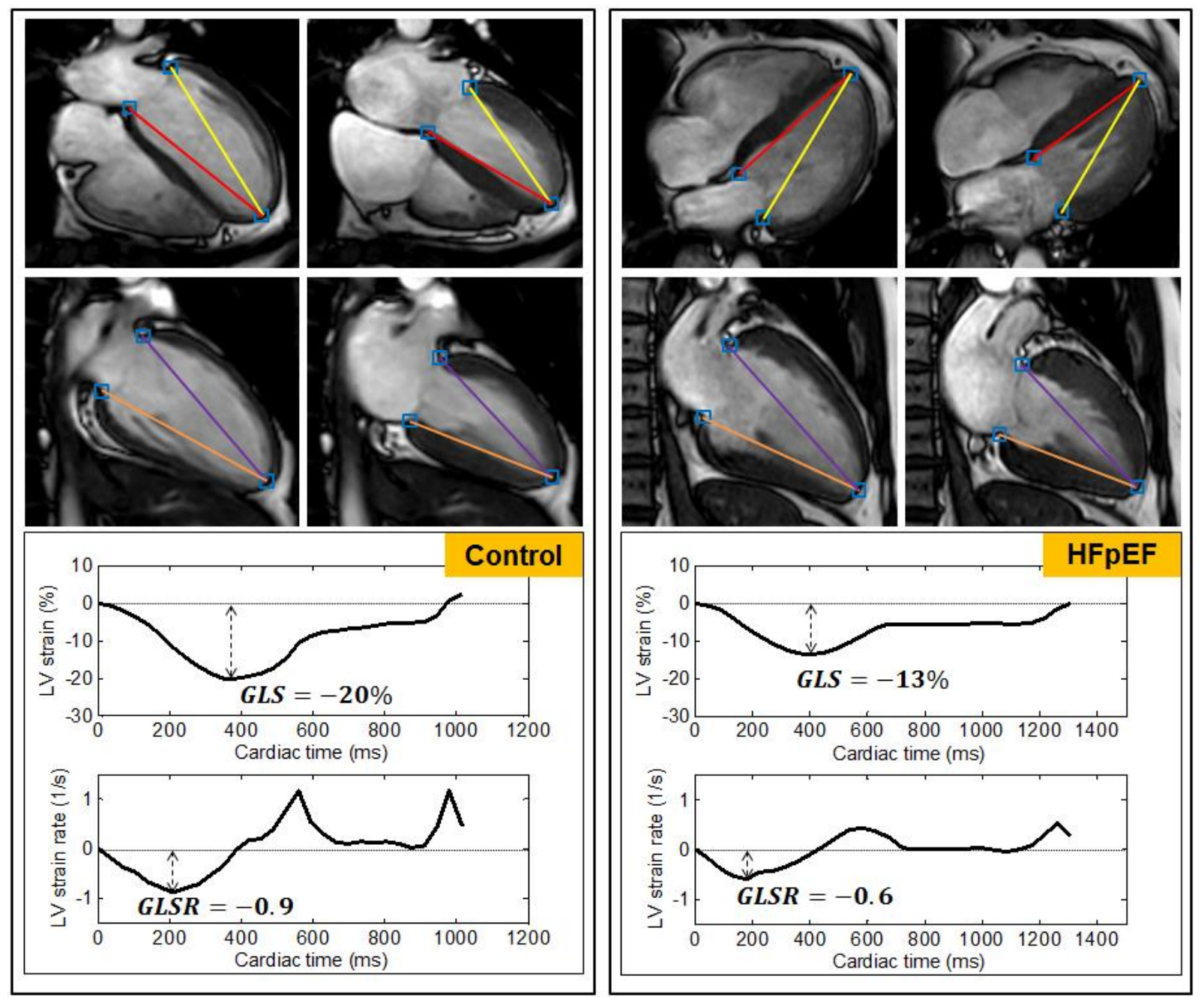

Figure 1. Fast left ventricular strain and strain rate measurement in four- and two-chamber views. Squares denote the atrioventricular junction points and apical epicardium that are tracked automatically throughout the cardiac cycle. The strain of each wall is calculated as a percentage using the presented strain formula. GLS represents the mean of all four measurements. GLS: global longitudinal strain. GLSR: global longitudinal strain rate.

Table 1. Comparison of fast left ventricular GLS and GSLR among subject groups.

\begin{tabular}{|c|c|c|c|c|}
\hline Parameters & $\begin{array}{l}\text { Controls } \\
(n=50)\end{array}$ & $\begin{array}{l}\text { HFpEF } \\
(n=30)\end{array}$ & $\begin{array}{l}\text { HFmrEF } \\
(n=30)\end{array}$ & $\begin{array}{l}\text { HFrEF } \\
(n=40)\end{array}$ \\
\hline GLS, \% & $-16.9 \pm 2.3$ & $-11.4 \pm 3.3^{*}$ & $-9.9 \pm 2.4^{*}$ & $-6.1 \pm 2.4^{\star} \# \$$ \\
\hline $\mathrm{GLSR}, \mathrm{s}^{-1}$ & $-0.85 \pm 0.15$ & $-0.63 \pm 0.17^{*}$ & $-0.57 \pm 0.17^{\star}$ & $-0.34 \pm 0.09^{*} \# \$$ \\
\hline LVEF, \% & $63.7 \pm 6.5$ & $56.4 \pm 4.5^{*}$ & $44.0 \pm 2.9^{*} \#$ & $25.8 \pm 8.3^{\star} \# \$$ \\
\hline
\end{tabular}

Data are represented as mean \pm SD. HFpEF: heart failure with preserved ejection fraction; HFmrEF: heart failure with midrange ejection fraction; HFrEF: heart failure with reduced ejection fraction; GLS: global longitudinal strain; GLSR: global longitudinal strain rate; LVEF: left ventricular ejection fraction. *significant difference compared to controls; \#significant difference compared to HFpEF; \$significant difference compared to HFmrEF. 